\title{
ON THE EXISTENCE OF SOLUTIONS OF A FERMAT-TYPE DIFFERENCE EQUATION
}

\author{
Nan Li \\ Shandong University, School of Mathematics, Jinan, Shandong, 250100, P. R. China \\ and University of Eastern Finland, Department of Physics and Mathematics \\ P. O. Box 111, 80101 Joensuu, Finland; nanli32787310@163.com
}

\begin{abstract}
The analogue of Fermat's last theorem for function fields has been investigated by many scholars recently, and Gundersen-Hayman [6] collected the best lower estimates that are known for $F_{C}(n)$, where $F_{C}(n)$ is the smallest positive integer $k$ such that the equation

$$
f_{1}^{n}+f_{2}^{n}+\ldots+f_{k}^{n}=1
$$

has a solution consisting of $k$ nonconstant functions $f_{1}, f_{2}, \ldots, f_{k}$ in $C$, and $C$ is the ring of meromorphic functions $M$, rational functions $R$, entire functions $E$ or polynomials $P$, respectively. In this paper, we investigate a difference analogue of this problem for the rings of $M, R, E, P$ with certain conditions, and obtain lower bounds for $G_{C}$, where $G_{C}(n)$ is the smallest positive integer $k$ such that the equation

$$
f_{1}(z) f_{1}(z+c) \cdots f_{1}(z+(n-1) c)+\ldots+f_{k}(z) f_{k}(z+c) \cdots f_{k}(z+(n-1) c)=1
$$
\end{abstract}

has a solution consisting of $k$ nonconstant functions $f_{1}, f_{2}, \ldots, f_{k}$ in $C$.

\section{Introduction}

According to the famous Fermat's last theorem, which was proved by Wiles [21] and by Taylor-Wiles [19], there do not exist nonzero rational numbers $x, y$, and an integer $n$, where $n \geq 3$, such that

$$
x^{n}+y^{n}=1 .
$$

There are natural analogues of Fermat's last theorem in complex function theory. For example, let $M, R, E$ and $P$ denote the rings of meromorphic functions, rational functions, entire functions and polynomials, respectively. Thus if $C$ is equal to $M$, $R, E$ or $P$, and $n$ is an integer satisfying $n \geq 2$, then $F_{C}(n)$ denotes the smallest positive integer $k$ such that the equation

$$
f_{1}^{n}+f_{2}^{n}+\cdots+f_{k}^{n}=1
$$

has a solution consisting of $k$ nonconstant functions $f_{1}, f_{2}, \ldots, f_{k}$ in $C$. Hence, the smallest $k$ depends on $n$.

Many scholars have investigated this and related problems, for details please see $[3,4,12,14,15,18,20,23,25]$ etc. Gundersen-Hayman [6] collected the best lower

doi:10.5186/aasfm.2015.4051

2010 Mathematics Subject Classification: Primary 30D35; Secondary 39A10.

Key words: Entire functions, meromorphic functions, Nevanlinna theory, Fermat-type equations.

This work was supported by the NNSF of China (No. 11171013 \& No. 11371225) and the Academy of Finland Grant \#268009. 
estimates that are known for every $n$ as follows:

$$
\begin{aligned}
& F_{P}(n)>1 / 2+\sqrt{n+1 / 4} ; \quad F_{R}(n)>\sqrt{n+1} ; \\
& F_{M}(n) \geq \sqrt{n+1} ; \quad F_{E}(n) \geq 1 / 2+\sqrt{n+1 / 4} .
\end{aligned}
$$

A natural difference analogue of the Taylor series expansion is the factorial series [17, p. 272], which suggests to consider the difference monomial $x(x-1) \cdots(x-n+1)$ as a discrete analogue of $x^{n}$. Similar correspondence occurs frequently in the theory of difference equations as well, and can be seen for example by comparing the Riccati equation and its difference analogue.

The purpose of this paper is to formulate and study a difference analogue of Fermat's last theorem for function fields $M, R, E, P$. We will consider the difference equation

$$
f_{1} \bar{f}_{1} \cdots \bar{f}_{1}^{[n-1]}+f_{2} \bar{f}_{2} \cdots \bar{f}_{2}^{[n-1]}+\cdots+f_{k} \bar{f}_{k} \cdots \bar{f}_{k}^{[n-1]}=1
$$

where $\bar{f}^{[i]}$ stands for $f(z+i c), c$ is a nonzero constant and $i$ is a positive integer, and denote by $G_{C}$ the smallest positive integer $k$ such that the equation (1.2) has a solution consisting of $k$ nonconstant functions $f_{1}, \ldots, f_{k}$ in $C$.

We need the following definition and notations in order to state our results.

Definition 1.1. [24] Let $f$ and $g$ be meromorphic functions and $a$ be a complex number. Let $z_{n}(n=1,2, \ldots)$ be zeros of $f-a$. If $z_{n}(n=1,2, \ldots)$ are also zeros of $g-a$ (ignoring multiplicity), we denote

$$
f=a \Rightarrow g=a \quad \text { or } \quad g=a \Leftarrow f=a .
$$

Let $\nu(n)$ be the multiplicity of the zero $z_{n}$. If $z_{n}(n=1,2, \ldots)$ are also $\nu(n)(n=$ $1,2, \ldots)$ multiple zeros of $g-a$ at least, we write

$$
f=a \rightarrow g=a \quad \text { or } \quad g=a \leftarrow f=a .
$$

If $f=a \rightleftharpoons g=a$, it is said that $f$ and $g$ share $a$ CM; If $f=a \Leftrightarrow g=a$, it is said that $f$ and $g$ share $a$ IM; If $f=a \rightarrow \bar{f}=a$ except for at most finitely many $a$-points of $f$, it is said that $a$ is an exceptional paired value of $f$ with the separation $c$ (as defined in [8]).

Let $\widetilde{M}$ be the collection of all nonconstant meromorphic functions of hyper-order $<1$ such that any finite collection $\left\{f_{1}, \ldots, f_{k}\right\} \subset \widetilde{M}$ satisfies the following properties

(i) $f_{i}$ and $1 / f_{j}(i, j=1, \ldots, k, i \neq j)$ have no common zeros;

(ii) $f_{i}=\infty \rightleftharpoons \bar{f}_{i}=\infty$ for all $i=1, \ldots, k$;

(iii) 0 is an exceptional paired value of $f_{i}$ for all $i=1, \ldots, k$.

In the case of meromorphic functions, compared to the lower bound of $F_{M}$, we obtain a corresponding result about $G_{\widetilde{M}}$.

Theorem 1.2. Let $n(\geq 2)$ be an integer. Then

$$
G_{\widetilde{M}}(n) \geq \sqrt{n+1} .
$$

Let $\widetilde{E}$ be the collection of all nonconstant entire functions of hyper-order $<1$ such that any finite collection $\left\{f_{1}, \ldots, f_{k}\right\} \subset \widetilde{E}$ satisfies the property that $f_{i}=0 \Rightarrow \bar{f}_{i}=0$ for all $i=1, \ldots, k$.

Particularly, for the case of entire functions, analogously to the lower bound of $F_{E}$, we give a better lower estimate for $G_{\widetilde{E}}$. 
Theorem 1.3. Let $n(\geq 2)$ be an integer. Then

$$
G_{\widetilde{E}}(n) \geq 1 / 2+\sqrt{n+1 / 4}
$$

The condition that hyper-order is less than 1 cannot be deleted. For example, take $f(z)=\exp \left\{e^{z}\right\}, c=i \pi$ and $n=2$. Since 0 and $\infty$ are Picard exceptional values of $f(z)$, they are automatically also exceptional paired values of $f(z)$. Moreover, the function also satisfies the conditions $f=0 \Rightarrow \bar{f}=0$ and $f=\infty \Rightarrow \bar{f}=\infty$. The hyper-order of $f(z)$ is 1 , and

$$
f(z) \cdot f(z+c)=\exp \left\{e^{z}\right\} \cdot \exp \left\{e^{z+i \pi}\right\}=\exp \left\{e^{z}\right\} \cdot \exp \left\{-e^{z}\right\}=1 .
$$

But $k=1$ is strictly less than $1 / 2+\sqrt{2+1 / 4}=\frac{3+1}{2}=2$ and $\sqrt{2+1}(>1)$.

The following example shows the sharpness of the bound of $G_{C}$, where $C$ is equal to $\widetilde{M}$ and $\widetilde{E}$.

Example 1.4. Let $c=2 \pi, f_{1}=\sin z$ and $f_{2}=\cos z$. Then $\bar{f}_{1}=\sin (z+2 \pi)=$ $\sin z \bar{f}_{2}=\cos (z+2 \pi)=\cos z$. Clearly $f_{i}(i=1,2)$ satisfy $f_{i}=0 \Rightarrow \bar{f}_{i}=0$ and

$$
f_{1} \bar{f}_{1}+f_{2} \bar{f}_{2}=\sin ^{2} z+\cos ^{2} z=1 .
$$

Also, 0 is an exceptional paired value of $f_{i}$ for $i=1,2$. Thus we have $G_{\widetilde{M}}(2) \leq 2$ and $G_{\widetilde{E}}(2) \leq 2$. On the other hand, by Theorems 1.2 and 1.3 , we have $G_{C}(n)>1$ for $C=\widetilde{M}, \widetilde{E}$. Therefore, $G_{\widetilde{M}}(2)=G_{\widetilde{E}}(2)=2$.

Let $\widetilde{R}$ be the collection of all nonconstant rational functions such that any finite collection $\left\{f_{1}, \ldots, f_{k}\right\} \subset \widetilde{R}$ satisfies the property that zeros and poles are of multiplicity positive integer multiple of $n$.

In the case of rational functions, compared to the lower bound for $F_{R}$, we get a corresponding estimate for $G_{\widetilde{R}}$.

Theorem 1.5. Let $n(\geq 2)$ be an integer. Then

$$
G_{\widetilde{R}}(n)>\sqrt{n+1}
$$

Let $\widetilde{P}$ be the collection of all nonconstant polynomial functions such that any finite collection $\left\{f_{1}, \ldots, f_{k}\right\} \subset \widetilde{P}$ satisfies the property that zeros are of multiplicity no less than $n$.

Also, as an analogue to the entire case, for the case of polynomials, we give a better lower estimate for $G_{\widetilde{P}}$.

Theorem 1.6. Let $n(\geq 2)$ be an integer. Then

$$
G_{\widetilde{P}}(n)>1 / 2+\sqrt{n+1 / 4}
$$

\section{Lemmas}

The following lemma on the growth of non-decreasing real-valued functions is a generalization of [9, Lemma 2.1]. It implies that shifting a characteristic or a counting function does not affect its growth significantly, provided that the hyper-order of the function is strictly less than one. 
Lemma 2.1. [10] Let $T:[0,+\infty) \rightarrow[0,+\infty)$ be a non-decreasing continuous function and let $s \in(0, \infty)$. If the hyper-order of $T$ is strictly less than one, i.e.,

$$
\limsup _{r \rightarrow \infty} \frac{\log \log T(r)}{\log r}=\varsigma<1
$$

and $\delta \in(0,1-\varsigma)$, then

$$
T(r+s)=T(r)+o\left(\frac{T(r)}{r^{\delta}}\right)
$$

where $r$ runs to infinity outside of a set of finite logarithmic measure.

Recently, Halburd, Korhonen and Tohge generalized the difference analogue of Logarithmic Derivative Lemma of meromorphic functions of finite order (see [2, 7]) into meromorphic functions of hyper-order strictly less than one.

Lemma 2.2. [10] Let $f$ be a non-constant meromorphic function, $\varepsilon>0$ and $c \in \mathbf{C}$. If the hyper-order of $T(r, f)$, i.e., $\sigma_{2}=\sigma_{2}(f)<1$, then

$$
m\left(r, \frac{f(z+c)}{f(z)}\right)=o\left(\frac{T(r, f)}{r^{1-\sigma_{2}-\varepsilon}}\right)
$$

for all $r$ outside of a set of finite logarithmic measure.

Throughout the remainder of the paper, we let $d(P)$ denote the degree of $P$, and let $\bar{d}(P)$ denote the number of distinct zeros of $P$, where $P(\not \equiv 0)$ is a polynomial. The following lemma is an application of Cartan's theorem.

Lemma 2.3. [6] Let $g_{1}, g_{2}, \cdots, g_{p}$ be linearly independent entire functions, where $p \geq 2$. Suppose that for each complex number $z$ we have

$$
\max \left\{\left|g_{1}(z)\right|,\left|g_{2}(z)\right|, \ldots,\left|g_{p}(z)\right|\right\}>0
$$

and set $g_{p+1}=g_{1}+g_{2}+\ldots+g_{p}$. We distinguish two cases.

(a) Suppose that all the quotients $g_{j} / g_{m}$ are rational functions. Then there exist polynomials $h_{1}, h_{2}, \ldots, h_{p+1}$, and an entire function $\phi$, such that

$$
g_{j}=h_{j} e^{\phi}, j=1,2, \ldots, p+1 .
$$

Then $h_{p+1}=h_{1}+h_{2}+\ldots+h_{p}$ and

$$
\max \left\{d\left(h_{1}\right), d\left(h_{2}\right), \ldots, d\left(h_{p}\right)\right\} \leq(p-1)\left\{\sum_{j=1}^{p+1} \bar{d}\left(h_{j}\right)-\frac{1}{2} p\right\} .
$$

In particular, if all the functions $g_{1}, g_{2}, \ldots, g_{p}$ are polynomials, then

$$
\max \left\{d\left(g_{1}\right), d\left(g_{2}\right), \ldots, d\left(g_{p}\right)\right\} \leq(p-1)\left\{\sum_{j=1}^{p+1} \bar{d}\left(g_{j}\right)-\frac{1}{2} p\right\} .
$$

(b) Suppose that at least one quotient $g_{j} / g_{m}$ is a transcendental function. Set

$$
N(r)=\sup _{1 \leq j \leq p+1} N\left(r, 0, g_{j}\right)
$$

Then

$$
\frac{N(r)}{\log r} \rightarrow \infty, \quad \text { as } r \rightarrow \infty
$$


and we have

$$
(1+o(1)) N(r) \leq(p-1) \sum_{j=1}^{p+1} \bar{N}\left(r, 0, g_{j}\right) \quad \text { as } r \rightarrow \infty \text { n.e. }
$$

where n.e. stands for nearly everywhere, which always means the inequality holds in the real axis outside of a finite logarithmic measure.

The following lemma tells us that if $f_{1}, \ldots, f_{k}$ is a collection of functions for which the minimum $G_{C}(n)=k$ is attained, then the corresponding terms on the left hand side of (1.2) must be linearly independent.

Lemma 2.4. Let $n(\geq 2)$ be an integer. Suppose the equation (1.2) has a solution consisting of $k$ nonconstant functions $f_{1}, \ldots, f_{k}$ in $C$. If $G_{C}(n)=k$, then the functions $f_{i} \cdots \bar{f}_{i}^{[n-1]}(i=1, \ldots, k)$ are linearly independent.

Proof. If $f_{i} \cdots \bar{f}_{i}^{[n-1]}(i=1, \ldots, k)$ are linearly dependent, then there exists complex constants $\alpha_{i}(i=1, \ldots, k)$, not all of which are 0 , such that

$$
\alpha_{1} f_{1} \cdots \bar{f}_{1}^{[n-1]}+\cdots+\alpha_{k} f_{k} \cdots \bar{f}_{k}^{[n-1]}=0 .
$$

Without loss of generality, we suppose that $\alpha_{i_{0}}\left(i_{0} \in\{1, \ldots, k\}\right) \neq 0$. Then from (2.6) we can get that

$$
\begin{aligned}
f_{i_{0}} \cdots \bar{f}_{i_{0}}^{[n-1]}= & \frac{\alpha_{1}}{\alpha_{i_{0}}} \cdot f_{1} \cdots \bar{f}_{1}^{[n-1]}+\cdots+\frac{\alpha_{i_{0}-1}}{\alpha_{i_{0}}} f_{i_{0}-1} \cdots \bar{f}_{i_{0}-1}^{[n-1]} \\
& +\frac{\alpha_{i_{0}+1}}{\alpha_{i_{0}}} f_{i_{0}+1} \cdots \bar{f}_{i_{0}+1}^{[n-1]}+\cdots+\frac{\alpha_{k}}{\alpha_{i_{0}}} \cdot f_{k} \cdots \bar{f}_{k}^{[n-1]} .
\end{aligned}
$$

Substituting (2.7) into (1.2), we get that

$$
\begin{aligned}
& \left(1+\frac{\alpha_{1}}{\alpha_{i_{0}}}\right) \cdot f_{1} \cdots \bar{f}_{1}^{[n-1]}+\cdots+\left(1+\frac{\alpha_{i_{0}-1}}{\alpha_{i_{0}}}\right) f_{i_{0}-1} \cdots \bar{f}_{i_{0}-1}^{[n-1]} \\
& +\left(1+\frac{\alpha_{i_{0}+1}}{\alpha_{i_{0}}}\right) f_{i_{0}+1} \cdots \bar{f}_{i_{0}+1}^{[n-1]}+\cdots+\left(1+\frac{\alpha_{k}}{\alpha_{i_{0}}}\right) f_{k} \cdots \bar{f}_{k}^{[n-1]}=1 .
\end{aligned}
$$

We set

$$
g_{j}=\left(1+\frac{\alpha_{j}}{\alpha_{i_{0}}}\right)^{1 / n} f_{j}, \quad j=1, \ldots, i_{0}-1, i_{0}+1, \ldots, k .
$$

Substituting (2.9) into (2.8), we then have

$g_{1} \cdots \bar{g}_{1}^{[n-1]}+g_{2} \cdots \bar{g}_{2}^{[n-1]}+\cdots+g_{i_{0}-1} \cdots \bar{g}_{i_{0}-1}^{[n-1]}+g_{i_{0}+1} \cdots \bar{g}_{i_{0}+1}^{[n-1]}+\cdots+g_{k} \cdots \bar{g}_{k}^{[n-1]}=1$, where $g_{j}\left(j=1, \ldots, i_{0}-1, i_{0}+1, \ldots, k\right)$ are non-constant functions in $C$. Thus we have $G_{C}(n)=k-1$, a contradiction with the assumption $k=G_{C}(n)$. Thus the functions $f_{i} \cdots \bar{f}_{i}^{[n-1]}(i=1, \ldots, k)$ are linearly independent.

\section{Proof of Theorem 1.3}

Suppose that $f_{1}, f_{2}, \ldots, f_{k}$ are nonconstant entire functions satisfying (1.2).

From the assumption $f_{i}=0 \Rightarrow \bar{f}_{i}=0$, we can get that $f_{i}(z)$ are transcendental. Since if there exists a $z_{0}$ such that $f_{i}\left(z_{0}\right)=0$, then $f_{i}\left(z_{0}+j c\right)=0$, where $i=1,2, \ldots, k$ and $j=1,2, \ldots$, so $f_{i}(z)$ has infinitely many zeros. Thus $f_{i}(z)$ is transcendental. If 
$f_{i}(z)$ has no zeros, then 0 is a Picard exceptional value of non-constant entire function $f_{i}(z)$, which implies that $f_{i}(z)$ is transcendental.

Firstly we prove that $k \geq 2$.

If $k=1$, then we have $f_{1} \cdots \bar{f}_{1}^{[n-1]}=1$. Since $f_{1}$ is a transcendental entire function, then from Lemma 2.2 and $\sigma_{2}\left(f_{1}\right)<1$, we have

$$
\begin{aligned}
n T\left(r, f_{1}\right) & =T\left(r, f_{1}^{n}\right)=T\left(r, \frac{1}{f_{1}^{n}}\right)+O(1)=T\left(r, \frac{\bar{f}_{1} \cdots \bar{f}_{1}^{[n-1]}}{f_{1}^{n-1}}\right)+O(1) \\
& =m\left(r, \frac{\bar{f}_{1} \cdots \bar{f}_{1}^{[n-1]}}{f_{1}^{n-1}}\right)+N\left(r, \frac{\bar{f}_{1} \cdots \bar{f}_{1}^{[n-1]}}{f_{1}^{n-1}}\right)+O(1) \\
& \leq N\left(r, \frac{1}{f_{1}^{n-1}}\right)+S\left(r, f_{1}\right) \leq(n-1) T\left(r, f_{1}\right)+S\left(r, f_{1}\right),
\end{aligned}
$$

a contradiction. Thus we have $k \geq 2$.

From Lemma 2.4, we know that if $f_{i} \cdots \bar{f}_{i}^{[n-1]}(i=1, \ldots, k)$ are linearly dependent, then we have $G_{E}(n) \neq k$. Since $G_{E}(n) \leq k$, so we have $G_{E}(n)<k$. This means that if $f_{i} \cdots \bar{f}_{i}^{[n-1]}(i=1, \ldots, k)$ are linearly dependent, then we can shorten the equation (1.2). Thus, in order to get the smallest $k$, from Lemma 2.4 we assume that the functions $f_{i} \cdots \bar{f}_{i}^{[n-1]}(i=1, \ldots, k)$ are linearly independent.

Next we prove that at least one $f_{i} \cdots \bar{f}_{i}^{[n-1]}$ is transcendental. Since $f_{i_{0}}$ is transcendental, also $f_{i_{0}}^{n}$ is transcendental. If $f_{i_{0}} \cdots \bar{f}_{i_{0}}^{[n-1]}$ is polynomial, we write $f_{i_{0}} \cdots \bar{f}_{i_{0}}^{[n-1]}=p(z)$. Thus from Lemma 2.2 and the fact that $f_{i_{0}}$ is a transcendental entire function with $\sigma_{2}\left(f_{i_{0}}\right)<1$, we get

$$
\begin{aligned}
n T\left(r, f_{i_{0}}\right)+S\left(r, f_{i_{0}}\right) & =T\left(r, f_{i_{0}}^{n}\right)+S\left(r, f_{i_{0}}\right)=T\left(r, \frac{p(z)}{f_{i_{0}}^{n}}\right) \\
& =T\left(r, \frac{\bar{f}_{i_{0}} \cdots \bar{f}_{i_{0}}^{[n-1]}}{f_{i_{0}}^{n-1}}\right) \leq(n-1) T\left(r, f_{i_{0}}\right)+S\left(r, f_{i_{0}}\right),
\end{aligned}
$$

a contradiction. So it follows that $f_{i_{0}} \cdots \bar{f}_{i_{0}}^{[n-1]}$ is transcendental. Dividing equation (1.2) by $f_{i_{0}} \cdots \bar{f}_{i_{0}}^{[n-1]}$, it therefore follows that at least one quotient

$$
f_{j} \cdots \bar{f}_{j}^{[n-1]} / f_{i_{0}} \cdots \bar{f}_{i_{0}}^{[n-1]}\left(j \neq i_{0}\right)
$$

must be transcendental. Next we apply Lemma 2.3 with $g_{j}=f_{j} \bar{f}_{j} \ldots \bar{f}_{j}^{[n-1]}, j=$ $1, \ldots, k$. Assumption (2.1) is satisfied for this set of functions, since otherwise we would get an immediate contradiction with (1.2). Then from Lemma 2.3, we find that

$$
(1+o(1)) N(r) \leq(k-1) \sum_{j=1}^{k} \bar{N}\left(r, 0, f_{j} \cdots \bar{f}_{j}^{[n-1]}\right) \quad \text { as } r \rightarrow \infty \text { n.e., }
$$

where $N(r)=\sup _{1 \leq j \leq k} N\left(r, 0, f_{j} \cdots \bar{f}_{j}^{[n-1]}\right)$. Since $\sigma_{2}\left(f_{i}\right)<1$, we have

$$
\limsup _{r \rightarrow \infty} \frac{\log \log \bar{N}\left(r, 0, f_{i}\right)}{\log r} \leq \sigma_{2}\left(f_{i}\right)<1 .
$$


By the assumption $f_{i}=0 \Rightarrow \bar{f}_{i}=0$, from (3.1), (3.2) and Lemma 2.1, we get

$$
\begin{aligned}
(1+o(1)) N(r) & \leq(k-1) \sum_{j=1}^{k} \bar{N}\left(r, 0, \bar{f}_{j}^{[n-1]}\right) \\
& \leq(k-1) \sum_{j=1}^{k} \bar{N}\left(r+(n-1)|c|, 0, f_{j}\right) \\
& =(k-1) \sum_{j=1}^{k}(1+o(1)) \bar{N}\left(r, 0, f_{j}\right) \\
& \leq(k-1) \frac{1}{n} \sum_{j=1}^{k}(1+o(1)) N\left(r, 0, f_{j} \cdots \bar{f}_{j}^{[n-1]}\right) \\
& \leq(k-1)(1+o(1)) \frac{k}{n} N(r), r \rightarrow \infty \text { n.e. }
\end{aligned}
$$

From (2.4) in Lemma 2.3, we have $N(r) \rightarrow \infty$ as $r \rightarrow \infty$. Hence from (3.3), we see that $n \leq k^{2}-k$. This proves Theorem 1.3.

\section{Proof of Theorem 1.2}

Suppose that each $f_{i}$ is a nonconstant meromorphic function. Next we prove that $f_{i}$ is transcendental.

Since zero is an exceptional paired value of $f_{i}$, then we get that either $f_{i}$ has no zeros or it has infinitely many zeros. From $f_{i}(z)$ and $f_{i}(z+c)$ share $\infty \mathrm{CM}$, we get that $f_{i}$ has no poles or it has infinitely many poles. Now we only need to consider the case when $f_{i}$ has no zeros and poles (in other cases, $f_{i}$ is transcendental obviously). But now, since 0 and $\infty$ are Picard exceptional values of a nonconstant meromorphic function $f_{i}$, it follows that $f_{i}$ is transcendental.

Next we prove that at least one $f_{i} \cdots{\overline{f_{i}}}^{[n-1]}$ is transcendental. Since $f_{i_{0}}$ is transcendental, then we have that $f_{i_{0}}^{n}$ is transcendental. Suppose that $f_{i_{0}} \cdots \bar{f}_{i_{0}}^{[n-1]}$ is polynomial, say, $p(z)$. Since $f_{i_{0}}$ is a transcendental meromorphic function with hyperorder less than 1 , then from Lemma 2.2 we get

$$
\begin{aligned}
n T\left(r, f_{i_{0}}\right)+S\left(r, f_{i_{0}}\right) & =T\left(r, f_{i_{0}}^{n}\right)+S\left(r, f_{i_{0}}\right)=T\left(r, \frac{p(z)}{f_{i_{0}}^{n}}\right) \\
& =N\left(r, \frac{\bar{f}_{i_{0}} \cdots \bar{f}_{i_{0}}^{[n-1]}}{f_{i_{0}}^{n-1}}\right)+S\left(r, f_{i_{0}}\right) .
\end{aligned}
$$

By the assumption that 0 is an exceptional paired value of $f_{i_{0}}$, we have

$$
N\left(r, \frac{\bar{f}_{i_{0}} \cdots \bar{f}_{i_{0}}^{[n-1]}}{f_{i_{0}}^{n-1}}\right) \leq N\left(r, \bar{f}_{i_{0}}\right)+\cdots+N\left(r, \bar{f}_{i_{0}}^{[n-1]}\right)+S\left(r, f_{i_{0}}\right) .
$$

From $\sigma_{2}\left(f_{i_{0}}\right)<1$, we have

$$
\limsup _{r \rightarrow \infty} \frac{\log \log N\left(r, f_{i_{0}}\right)}{\log r} \leq \sigma_{2}\left(f_{i_{0}}\right)<1 .
$$


Thus by a simple observation, Lemma 2.1, (4.1) and (4.2) we get

$$
\begin{aligned}
n T\left(r, f_{i_{0}}\right) & \leq N\left(r+|c|, f_{i_{0}}\right)+\cdots+N\left(r+(n-1)|c|, f_{i_{0}}\right)+S\left(r, f_{i_{0}}\right) \\
& \leq N\left(r, f_{i_{0}}\right)+\cdots+N\left(r, f_{i_{0}}\right)+S\left(r, f_{i_{0}}\right) \\
& \leq(n-1) T\left(r, f_{i_{0}}\right)+S\left(r, f_{i_{0}}\right),
\end{aligned}
$$

which yields a contradiction. So we have that $f_{i_{0}} \cdots \bar{f}_{i_{0}}^{[n-1]}$ is transcendental.

As in the proof of Theorem 1.3, by dividing with $f_{i_{0}} \cdots \bar{f}_{i_{0}}^{[n-1]}$ on both sides of (1.2), we obtain that at least one quotient $\frac{f_{j} \cdots \bar{f}_{j}^{[n-1]}}{f_{i_{0}} \cdots \bar{f}_{i_{0}}^{n-1]}}\left(j \neq i_{0}\right)$ must be transcendental.

For every meromorphic function $f_{i}(i=1,2, \ldots, k)$ with $\sigma_{2}\left(f_{i}\right)<1$, there exists linearly independent entire functions $g_{i}$ and $h_{i}$ with no common zeros such that

$$
f_{i}(z)=\frac{g_{i}(z)}{h_{i}(z)}
$$

Since entire functions $g_{i}$ and $h_{i}$ have no common zeros, we have that $g_{i}$ and $f_{i}$ have the same zeros and that $h_{i}$ and $1 / f_{i}$ have the same zeros. Thus we have

$$
\lambda_{2}\left(g_{i}\right)=\limsup _{r \rightarrow \infty} \frac{\log ^{+} \log ^{+} N\left(r, 0, g_{i}\right)}{\log r}=\lambda_{2}\left(f_{i}\right) \leq \sigma_{2}\left(f_{i}\right)<1
$$

and

$$
\lambda_{2}\left(h_{i}\right)=\limsup _{r \rightarrow \infty} \frac{\log ^{+} \log ^{+} N\left(r, 0, h_{i}\right)}{\log r}=\lambda_{2}\left(\frac{1}{f_{i}}\right) \leq \sigma_{2}\left(f_{i}\right)<1 .
$$

Substituting (4.3) into (1.2), and multiplying both sides by $h_{1} \cdots \bar{h}_{1}^{[n-1]} h_{2} \cdots \bar{h}_{2}^{[n-1]}$ $\cdots h_{k} \cdots \bar{h}_{k}^{[n-1]}$, we have

$$
l_{1}+l_{2}+\cdots+l_{k}=l_{k+1} \text {, }
$$

where

$$
\begin{aligned}
& l_{1}=g_{1} \cdots \bar{g}_{1}^{[n-1]} h_{2} \cdots \bar{h}_{2}^{[n-1]} \cdots h_{k} \cdots \bar{h}_{k}^{[n-1]}, \\
& l_{2}=g_{2} \cdots \bar{g}_{2}^{[n-1]} h_{1} \cdots \bar{h}_{1}^{[n-1]} h_{3} \cdots \bar{h}_{3}^{[n-1]} \cdots h_{k} \cdots \bar{h}_{k}^{[n-1]}, \\
& \cdots \\
& l_{k}=g_{k} \cdots \bar{g}_{k}^{[n-1]} h_{1} \cdots \bar{h}_{1}^{[n-1]} h_{2} \cdots \bar{h}_{2}^{[n-1]} \cdots h_{k-1} \cdots \bar{h}_{k-1}^{[n-1]}, \\
& l_{k+1}=h_{1} \cdots \bar{h}_{1}^{[n-1]} \cdots h_{k} \cdots \bar{h}_{k}^{[n-1]} .
\end{aligned}
$$

We now define a function $d(z)$ as follows.

Case 1. Suppose that $l_{i}(i=1, \ldots, k)$ have infinitely many non-zero common zeros. Let $\left\{a_{n}\right\}$ be the non-zero common zeros of $l_{i}(i=1, \ldots, k)$ such that $\left|a_{n}\right| \rightarrow \infty$ (otherwise, if $\left|a_{n}\right|<M$ as $n \rightarrow \infty$, it would follow that $l_{i} \equiv 0$ ). If $\left\{p_{n}\right\}$ is any sequence of non-negative integers such that for all $r>0$,

$$
\sum_{n=1}^{\infty}\left(r /\left|a_{n}\right|\right)^{1+p_{n}}<\infty
$$


then from the Weierstrass theorem, we have that the function

$$
d(z)=\prod_{n=1}^{\infty} E_{p_{n}}\left(z / a_{n}\right)
$$

is entire with zeros only at points $a_{n}$, where

$$
E_{p_{n}}= \begin{cases}(1-z), & \text { if } p_{n}=0, \\ (1-z) \exp \left(\frac{z^{1}}{1}+\frac{z^{2}}{2}+\cdots+\frac{z^{p_{n}}}{p_{n}}\right), & \text { otherwise. }\end{cases}
$$

If the number $z_{0}$ occurs in the sequence $\left\{a_{n}\right\}$ exactly $m$ times, then function $d(z)$ has a zero at $z=z_{0}$ of multiplicity $m$.

Case 2. Suppose that $l_{i}(i=1, \ldots, k)$ have finitely many non-zero common zeros. We set $d(z)=\prod_{n=1}^{n_{0}}\left(z-a_{n}\right)$.

Suppose also that $l_{i}(i=1, \ldots, k)$ have a common zero at $z=0$ of order $m \geq 0$ (a zero of order $m=0$ at $z=0$ means $f(0) \neq 0$ ).

With $d(z)$ defined according to Cases 1 and 2, let

$$
\widetilde{l}_{i}(z)=\frac{l_{i}(z)}{z^{m} d(z)}, \quad i=1, \ldots, k+1 .
$$

Then for each complex number $z \in \mathbf{C}$, we have $\max \left\{\left|\widetilde{l}_{1}(z)\right|,\left|\widetilde{l}_{2}(z)\right|, \ldots,\left|\widetilde{l}_{k}(z)\right|\right\}>0$ and

$$
\widetilde{l}_{1}+\widetilde{l}_{2}+\cdots+\widetilde{l}_{k}=\widetilde{l}_{k+1}
$$

where $\widetilde{l}_{i}(z)(i=1, \ldots, k+1)$ are entire functions.

From (4.7), (4.8) and (4.3), we obtain that

$$
\frac{\widetilde{l}_{j}}{\widetilde{l}_{i_{0}}}=\frac{g_{j} \cdots \bar{g}_{j}^{[n-1]}}{h_{j} \cdots \bar{h}_{j}^{[n-1]}} \cdot \frac{h_{i_{0}} \cdots \bar{h}_{i_{0}}^{[n-1]}}{g_{i_{0}} \cdots \bar{g}_{i_{0}}^{[n-1]}}=\frac{f_{j} \cdots \bar{f}_{j}^{[n-1]}}{f_{i_{0}} \cdots \bar{f}_{i_{0}}^{[n-1]}},
$$

$j=1, \ldots, i_{0}-1, i_{0}+1, \ldots, n$. In the beginning of the proof, we obtained that at least one quotient $\frac{f_{j} \cdots \bar{f}_{j}^{[n-1]}}{f_{i_{0}} \cdots \bar{f}_{i_{0}}^{n-1]}}\left(j \neq i_{0}\right)$ must be transcendental, thus it follows from (4.10) that at least one $\frac{\widetilde{l_{j}}}{\widetilde{l}_{i_{0}}}\left(j \neq i_{0}\right)$ must be transcendental.

Next, we prove $k \geq 2$. If not, $k=1$, then we have $\widetilde{l}_{1}=\widetilde{l}_{2}$, thus $l_{1}=l_{2}$, i.e. $f_{1} \cdots \bar{f}_{1}^{[n-1]}=1$. Since $\sigma_{2}\left(f_{1}\right)<1$, we have

$$
\limsup _{r \rightarrow \infty} \frac{\log \log N\left(r, f_{1}\right)}{\log r} \leq \sigma_{2}\left(f_{1}\right)<1 .
$$

Combining this with the fact that 0 is an exceptional paired value of transcendental meromorphic function $f_{1}$, from Lemma 2.2 and Lemma 2.1, we have

$$
\begin{aligned}
n T\left(r, f_{1}\right) & =T\left(r, \frac{1}{f_{1}^{n}}\right)+O(1)=T\left(r, \frac{\bar{f}_{1} \cdots \bar{f}_{1}^{[n-1]}}{f_{1}^{n-1}}\right)+O(1) \\
& \leq N\left(r, \frac{\bar{f}_{1}}{f_{1}}\right)+\cdots+N\left(r, \frac{\bar{f}_{1}^{[n-1]}}{f_{1}}\right)+S\left(r, f_{1}\right)
\end{aligned}
$$




$$
\begin{aligned}
& \leq N\left(r, \bar{f}_{1}\right)+\cdots+N\left(r, \bar{f}_{1}^{[n-1]}\right)+S\left(r, f_{1}\right) \\
& \leq(n-1) N\left(r, f_{1}\right)+S\left(r, f_{1}\right) \leq(n-1) T\left(r, f_{1}\right)+S\left(r, f_{1}\right),
\end{aligned}
$$

a contradiction. Thus we have $k \geq 2$.

In order to get the smallest $k$, we assume that the functions $\widetilde{l}_{1}, \ldots, \widetilde{l}_{k}$ are linearly independent. Otherwise, if the functions $\widetilde{l}_{1}, \ldots, \widetilde{l}_{k}$ are linearly dependent, then there exists $\alpha_{1}, \ldots, \alpha_{k}$, not all of which are zeros, such that

$$
\alpha_{1} \widetilde{l}_{1}+\cdots+\alpha_{k} \widetilde{l}_{k}=0
$$

Without loss of generality, we suppose that $\alpha_{i_{0}} \neq 0$. Then from (4.11), we have

$$
\widetilde{l}_{i_{0}}=\frac{\alpha_{1}}{\alpha_{i_{0}}} \widetilde{l}_{1}+\cdots+\frac{\alpha_{i_{0}-1}}{\alpha_{i_{0}}} \widetilde{l}_{i_{0}-1}+\frac{\alpha_{i_{0}+1}}{\alpha_{i_{0}}} \widetilde{l}_{i_{0}+1}+\cdots+\frac{\alpha_{k}}{\alpha_{i_{0}}} \widetilde{l}_{k}
$$

Dividing (4.12) by $\widetilde{l}_{k+1}$ on both sides, from (4.7) and (4.8) we have that

$$
\begin{aligned}
f_{i_{0}} \cdots \bar{f}_{i_{0}}^{[n-1]}= & \frac{\alpha_{1}}{\alpha_{i_{0}}} f_{1} \cdots \bar{f}_{1}^{[n-1]}+\cdots+\frac{\alpha_{i_{0}-1}}{\alpha_{i_{0}}} f_{i_{0}-1} \cdots \bar{f}_{i_{0}-1}^{[n-1]} \\
& +\frac{\alpha_{i_{0}+1}}{\alpha_{i_{0}}} f_{i_{0}+1} \cdots \bar{f}_{i_{0}+1}^{[n-1]}+\cdots+\frac{\alpha_{k}}{\alpha_{i_{0}}} f_{k} \cdots \bar{f}_{k}^{[n-1]} .
\end{aligned}
$$

This gives that $f_{1} \cdots \bar{f}_{1}^{[n-1]}, \ldots, f_{k} \cdots \bar{f}_{k}^{[n-1]}$ are linearly dependent, which contradicts with Lemma 2.4. So in order to get the smallest $k$, we assume that $\widetilde{l}_{1}, \ldots, \widetilde{l}_{k}$ are linearly independent.

Thus we can apply Lemma 2.3 to (4.9), and this yields

$$
(1+o(1)) N(r) \leq(k-1) \sum_{j=1}^{k+1} \bar{N}\left(r, 0, \widetilde{l}_{j}\right) \text { as } r \rightarrow \infty \text { n.e., }
$$

where $N(r)=\sup _{1 \leq j \leq k+1} N\left(r, 0, \widetilde{l}_{j}\right)$. Since $f_{i}=g_{i} / h_{i}$, where $g_{i}$ and $h_{i}$ are entire functions with no common zeros, from $f_{i}$ and $\bar{f}_{i}$ share $\infty \mathrm{CM}$, we get that $h_{i}$ and $\bar{h}_{i}$ share 0 CM. Also, from 0 is an exceptional paired value of $f_{i}$, we get that 0 is an exceptional paired value of $g_{i}$.

Next we analyze the multiplicity of zeros of $h_{1} \cdots \bar{h}_{1}^{[n-1]} \cdots h_{j-1} \cdots \bar{h}_{j-1}^{[n-1]} h_{j+1} \cdots$ $\left.\bar{h}_{j+1}^{[n-1]} \cdots h_{k} \cdots \bar{h}_{k}^{[n-1]} /\left(z^{m} d(z)\right)\right)$. From (4.7) and (4.6), we know that all the zeros of $z^{m} d(z)$ arise from the zeros of at least one $h_{i}(i=1, \ldots, k)$. Since $f_{i}$ and $1 / f_{j}$ do not have common zeros, we have that $g_{i}$ and $h_{j}$ do not have common zeros. Since $h_{i}$ and $\bar{h}_{i}$ share $0 \mathrm{CM}$, so we have that $g_{i} \cdots \bar{g}_{i}^{[n-1]}$ and $h_{j} \cdots \bar{h}_{j}^{[n-1]}$ do not have common zeros. Thus $g_{i} \cdots \bar{g}_{i}^{[n-1]}$ and $h_{1} \cdots \bar{h}_{1}^{[n-1]} \cdots h_{k} \cdots \bar{h}_{k}^{[n-1]}$ do not have common zeros. So we have that the common zeros of $l_{1}, \ldots, l_{k}$ (i.e., the zeros of $z^{m} d(z)$ ) arise from the common zeros of

$$
h_{1} \cdots \bar{h}_{1}^{[n-1]} \cdots h_{i-1} \cdots \bar{h}_{i-1}^{[n-1]} h_{i+1} \cdots \bar{h}_{i+1}^{[n-1]} \cdots h_{k} \cdots \bar{h}_{k}^{[n-1]} \quad(i=1, \ldots, k) .
$$


From $h_{i}$ and $\bar{h}_{i}$ share $0 \mathrm{CM}$, thus we have

$$
\begin{aligned}
& N\left(r, 0, \frac{h_{1} \cdots \bar{h}_{1}^{[n-1]} \cdots h_{i-1} \cdots \bar{h}_{i-1}^{[n-1]} h_{i+1} \cdots \bar{h}_{i+1}^{[n-1]} \cdots h_{k} \cdots \bar{h}_{k}^{[n-1]}}{z^{m} d(z)}\right) \\
& \geq n \bar{N}\left(r, 0, \frac{h_{1} \cdots \bar{h}_{1}^{[n-1]} \cdots h_{i-1} \cdots \bar{h}_{i-1}^{[n-1]} h_{i+1} \cdots \bar{h}_{i+1}^{[n-1]} \cdots h_{k} \cdots \bar{h}_{k}^{[n-1]}}{z^{m} d(z)}\right),
\end{aligned}
$$

i.e.,

$$
N\left(r, 0, \widetilde{l}_{i} / g_{i} \cdots \bar{g}_{i}^{[n-1]}\right) \geq n \bar{N}\left(r, 0, \widetilde{l}_{i} / g_{i} \cdots \bar{g}_{i}^{[n-1]}\right) .
$$

From above analysis, we also have

$$
\begin{aligned}
N\left(r, 0, \widetilde{l}_{k+1}\right) & =N\left(r, 0, h_{1} \cdots \bar{h}_{1}^{[n-1]} \cdots h_{k} \cdots \bar{h}_{k}^{[n-1]} /\left(z^{m} d(z)\right)\right) \\
& \geq n \bar{N}\left(r, 0, h_{1} \cdots \bar{h}_{1}^{[n-1]} \cdots h_{k} \cdots \bar{h}_{k}^{[n-1]} /\left(z^{m} d(z)\right)\right) \\
& =n \bar{N}\left(r, 0, \widetilde{l}_{k+1}\right) .
\end{aligned}
$$

From (4.14), (4.15), (4.13) and 0 is the exceptional paired value of $g_{i}$, we obtain that

$$
\begin{aligned}
& (1+o(1)) N(r) \leq(k-1)\left(\sum_{j=1}^{k} \bar{N}\left(r, 0, \frac{\widetilde{l}_{j}}{g_{j} \cdots \bar{g}_{j}^{[n-1]}} \cdot g_{j} \cdots \bar{g}_{j}^{[n-1]}\right)+\bar{N}\left(r, 0, \widetilde{l}_{k+1}\right)\right) \\
& \leq(k-1)\left(\sum_{j=1}^{k}\left(\bar{N}\left(r, 0, g_{j} \cdots \bar{g}_{j}^{[n-1]}\right)+\bar{N}\left(r, 0, \frac{\widetilde{l}_{j}}{g_{j} \cdots \bar{g}_{j}^{[n-1]}}\right)\right)+\bar{N}\left(r, 0, \widetilde{l}_{k+1}\right)\right) \\
& \leq(k-1)\left(\sum_{j=1}^{k}\left(\bar{N}\left(r, 0, \bar{g}_{j}^{[n-1]}\right)+\frac{1}{n} N\left(r, 0, \frac{\widetilde{l}_{j}}{g_{j} \cdots \bar{g}_{j}^{[n-1]}}\right)\right)+\frac{1}{n} N\left(r, 0, \widetilde{l}_{k+1}\right)\right) \\
& \leq(k-1)\left(\sum_{j=1}^{k}\left(\bar{N}\left(r+(n-1)|c|, 0, g_{j}\right)+\frac{1}{n} N\left(r, 0, \frac{\widetilde{l}_{j}}{g_{j} \cdots \bar{g}_{j}^{[n-1]}}\right)\right)+\frac{1}{n} N\left(r, 0, \widetilde{l}_{k+1}\right)\right) .
\end{aligned}
$$

From (4.4), we have

$$
\limsup _{r \rightarrow \infty} \frac{\log \log \bar{N}\left(r, 0, g_{i}\right)}{\log r} \leq \lambda_{2}\left(g_{i}\right)<1 .
$$

Thus by Lemma 2.1, and from 0 is an exceptional paired value of $g_{i}$, we have

$$
\begin{aligned}
& (1+o(1)) N(r) \\
& \leq(k-1)\left(\sum_{j=1}^{k}\left(\bar{N}\left(r, 0, g_{j}\right)+\frac{1}{n} N\left(r, 0, \frac{\widetilde{l}_{j}}{g_{j} \cdots \bar{g}_{j}^{[n-1]}}\right)\right)+\frac{1}{n} N\left(r, 0, \widetilde{l}_{k+1}\right)\right) \\
& \leq(k-1) \frac{1}{n}\left(\sum_{j=1}^{k}\left(N\left(r, 0, g_{j} \cdots \bar{g}_{j}^{[n-1]}\right)+N\left(r, 0, \frac{\widetilde{l}_{j}}{g_{j} \cdots \bar{g}_{j}^{[n-1]}}\right)\right)\right. \\
& \left.+N\left(r, 0, \widetilde{l}_{k+1}\right)\right) .
\end{aligned}
$$


Since $\widetilde{l}_{j}, g_{j}$ and $\frac{\widetilde{l}_{j}}{g_{j} \cdots \bar{g}_{j}^{n-1]}}$ are all entire functions, we have

$$
N\left(r, 0, g_{j} \cdots \bar{g}_{j}^{[n-1]}\right)+N\left(r, 0, \frac{\widetilde{l}_{j}}{g_{j} \cdots \bar{g}_{j}^{[n-1]}}\right)=N\left(r, 0, \widetilde{l}_{j}\right) .
$$

Therefore, combining (4.16) with (4.17), we have

$$
(1+o(1)) N(r) \leq(k-1) \frac{1}{n}\left(\sum_{j=1}^{k+1} N\left(r, 0, \widetilde{l}_{j}\right)\right) \leq(k-1) \frac{1}{n}(k+1) N(r),
$$

as $r \rightarrow \infty$ n.e. From Lemma 2.3, we get that $N(r) \rightarrow \infty$ as $r \rightarrow \infty$ n.e. Combining this with (4.18), we obtain that $n \leq k^{2}-1$.

\section{Proof of Theorem 1.6}

Suppose that $f_{1}, \ldots, f_{k}$ are nonconstant polynomial functions whose zeros are of multiplicity no less than $n$.

First, we prove that $k>1$. If $k=1$, then from (1.2) we have that

$$
\operatorname{deg}\left(f_{1} \ldots \bar{f}_{1}^{[n-1]}\right)=n \operatorname{deg}\left(f_{1}\right)=\operatorname{deg} 1=0,
$$

a contradiction. Thus we have $k \geq 2$.

In order to get the smallest $k$, we can assume using Lemma 2.4 that the polynomial functions $f_{i} \ldots \bar{f}_{i}^{[n-1]}(i=1, \ldots, k)$ are linearly independent.

Obviously, from $(1.2)$ we can get that $f_{i} \ldots \bar{f}_{i}^{[n-1]}(i=1, \ldots, k)$ do not have common zeros. Set $d=\max \left\{d\left(f_{1}\right), \ldots, d\left(f_{k}\right)\right\}$. Then for $i=1, \ldots, n-1$, we have

$$
\max \left\{d\left(\bar{f}_{1}^{[i]}\right), \ldots, d\left(\bar{f}_{k}^{[i]}\right)\right\}=d,
$$

and $d>0$. Choosing $g_{j}=f_{j} \cdots \bar{f}_{j}^{[n-1]}(j=1, \ldots, k)$ and $g_{k+1}=1$, we have that the assumption (2.1) is satisfied. Thus, it follows by (2.3) in Lemma 2.3 that

$$
\begin{aligned}
n \cdot d & =n \cdot \max \left\{d\left(f_{1}\right), \ldots, d\left(f_{k}\right)\right\}=\max \left\{d\left(f_{1} \ldots \bar{f}_{1}^{[n-1]}\right), \ldots, d\left(f_{k} \ldots f_{k}^{[n-1]}\right)\right\} \\
& =\max \left\{d\left(g_{1}\right), \ldots, d\left(g_{k}\right)\right\} \leq(k-1)\left\{\sum_{j=1}^{k} \bar{d}\left(g_{j}\right)-\frac{1}{2} k\right\} \\
1) & =(k-1)\left\{\sum_{j=1}^{k} \bar{d}\left(f_{j} \ldots \bar{f}_{j}^{[n-1]}\right)-\frac{1}{2} k\right\} .
\end{aligned}
$$

Since the zeros of $f_{1}, \ldots, f_{k}$ are of multiplicity no less than $n$, so by (5.1) we have

$$
\begin{aligned}
n \cdot d & \leq(k-1)\left\{\sum_{j=1}^{k}\left(\bar{d}\left(f_{j}\right)+\ldots+\bar{d}\left(\bar{f}_{j}^{[n-1]}\right)\right)-\frac{1}{2} k\right\} \\
& \leq(k-1)\left\{\sum_{j=1}^{k} \frac{1}{n}\left(d\left(f_{j}\right)+\ldots+d\left(\bar{f}_{j}^{[n-1]}\right)\right)-\frac{1}{2} k\right\} \\
& \leq(k-1)\left\{k \cdot d-\frac{1}{2} k\right\}<(k-1) k \cdot d,
\end{aligned}
$$


which yields $n<k^{2}-k$.

\section{Proof of Theorem 1.5}

Suppose that $f_{1}, \ldots, f_{k}$ are nonconstant rational functions whose zeros and poles are of multiplicity positive integer multiple of $n$, where $n(\geq 2)$ is a positive integer.

Suppose that at least one $f_{j}$ is not polynomial. We set

$$
f_{j}=\frac{P_{j}}{Q_{j}}
$$

where $P_{j}$ and $Q_{j}$ are polynomials without common zeros, and the zeros of $P_{j}$ and $Q_{j}$ are of multiplicity positive integer multiple of $n$. Substituting (6.1) into (1.2), then we obtain that

$$
R_{1}+R_{2}+\cdots+R_{k}=R_{k+1}
$$

where

$$
R_{i}=P_{i} \cdots \bar{P}_{i}^{[n-1]} Q_{1} \cdots \bar{Q}_{1}^{[n-1]} \cdots Q_{i-1} \cdots \bar{Q}_{i-1}^{[n-1]} Q_{i+1} \cdots \bar{Q}_{i+1}^{[n-1]} \cdots Q_{k} \cdots \bar{Q}_{k}^{[n-1]}
$$

$i=1, \ldots, k$, and

$$
R_{k+1}=Q_{1} \cdots \bar{Q}_{1}^{[n-1]} \cdots Q_{k} \cdots \bar{Q}_{k}^{[n-1]} .
$$

If $R_{1}, \ldots, R_{k}$ have common zeros $\left\{z_{i}\right\}$ with multiplicities $m_{i}$, then the number of common zeros must be finite. We let $d(z)=\prod\left(z-z_{i}\right)^{m_{i}}$, then $d(z)$ is a polynomial. By assumption, it follows that $n \mid m_{i}$.

We set

$$
\widetilde{R}_{i}=\frac{R_{i}}{d}, \quad i=1, \ldots, k+1
$$

Then we have that $\widetilde{R}_{1}, \ldots, \widetilde{R}_{k+1}$ are polynomials. Combining (6.2) with (6.5) we have

$$
\widetilde{R}_{1}+\widetilde{R}_{2}+\cdots+\widetilde{R}_{k}=\widetilde{R}_{k+1},
$$

where $\widetilde{R}_{1}, \ldots, \widetilde{R}_{k}$ have no common zeros. In addition, by $n \mid m_{i}$ and the assumption that the zeros of $P_{j}$ and $Q_{j}$ are of multiplicity positive integer multiple of $n$, it follows from (6.3), (6.4) and (6.5) that

$$
N\left(r, \frac{1}{\widetilde{R}_{i}}\right) \geq n \bar{N}\left(r, \frac{1}{\widetilde{R}_{i}}\right), \quad \text { for } i=1, \ldots, k+1 .
$$

Next, we prove that $k>1$. If $k=1$, then we have $R_{1}=R_{2}$. From (6.3) and (6.4), it follows that

$$
P_{1} \cdots \bar{P}_{1}^{[n-1]} \equiv Q_{1} \cdots \bar{Q}_{1}^{[n-1]}
$$

Since $P_{1}$ and $Q_{1}$ have no common zeros, and zeros of $\bar{P}_{1}^{[j]}$ and $\bar{Q}_{1}^{[j]}$ are zeros of $P_{1}$ and $Q_{1}$ after shifting with $j c$ in the same direction respectively, it follows that (6.8) yields a contradiction.

In order to get the smallest $k$, we assume that the functions $\widetilde{R}_{1}, \ldots, \widetilde{R}_{k}$ are linearly independent. Otherwise, if the functions $\widetilde{R}_{1}, \ldots, \widetilde{R}_{k}$ are linearly dependent, then there exists $\alpha_{1}, \ldots, \alpha_{k}$, not all of which are zeros, such that

$$
\alpha_{1} \widetilde{R}_{1}+\ldots+\alpha_{k} \widetilde{R}_{k}=0 .
$$


Following a similar argument as in the proof of Theorem 1.2, we get that $f_{1} \cdots \bar{f}_{1}^{[n-1]}$, $\ldots, f_{k} \cdots \bar{f}_{k}^{[n-1]}$ are linearly dependent, which contradicts with the Lemma 2.4. So in order to get the smallest $k$, we assume that $\widetilde{R}_{1}, \ldots, \widetilde{R}_{k}$ are linearly independent.

We set $d^{\prime}=\max \left\{d\left(\widetilde{R}_{1}\right), \ldots, d\left(\widetilde{R}_{k}\right)\right\}$. Then from (6.6), we know that

$$
d\left(\widetilde{R}_{k+1}\right)=d\left(\widetilde{R}_{1}+\widetilde{R}_{2}+\cdots+\widetilde{R}_{k}\right) \leq \max \left\{d\left(\widetilde{R}_{1}\right), \ldots, d\left(\widetilde{R}_{k}\right)\right\}=d^{\prime} .
$$

Thus combining this with (6.7), we can apply Lemma 2.3 (a) to (6.6), and obtain

$$
d^{\prime} \leq(k-1)\left\{\sum_{j=1}^{k+1} \bar{d}\left(\widetilde{R}_{j}\right)-\frac{1}{2} k\right\} \leq(k-1)\left\{(k+1) \frac{d^{\prime}}{n}-\frac{1}{2} k\right\}<\left(k^{2}-1\right) \frac{d^{\prime}}{n},
$$

which yields $n<k^{2}-1$.

Acknowledgments. The author would like to express sincere thanks to her supervisor in UEF - Prof. Risto Korhonen for many valuable suggestions to the present paper. The author also would like to thank the referee for his/her valuable suggestions.

\section{References}

[1] Cartan, H.: Sur lés zeros des combinaisons linéaires de p fonctions holomorphes données. Mathematica Cluj 7, 1933, 5-31.

[2] Chiang, Y. M., and S. J. Feng: On the growth of logarithmic differences, difference quotients and logarithmic derivatives of meromorphic functions. - Trans. Amer. Math. Soc. 361:7, 2009, $3767-3791$.

[3] Fujimoto, H.: On meromorphic maps into the complex projective space. - J. Math. Soc. Japan 26, 1974, 272-288.

[4] Green, M. L.: Some Picard theorems for holomorphic maps to algebraic varieties. - Amer. J. Math. 97, 1975, 43-75.

[5] Gross, F.: On the equation $f^{n}+g^{n}=1$. - Bull. Amer. Math. Soc. 72, 1966, 86-88. Correction: ibid. $72,1966,576$.

[6] Gundersen, G. G., and W. K. Hayman: The strength of Cartan's version of Nevanlinna theory. - Bull. London Math. Soc. 36:4, 2004, 433-454.

[7] Halburd, R. G., and R. J. Korhonen: Difference analogue of the lemma on the logarithmic derivative with applications to difference equations. - J. Math. Anal. Appl. 314:2, 2006, 477487.

[8] Halburd, R. G., and R. J. Korhonen: Nevanlinna theory for the difference operator. - Ann. Acad. Sci. Fenn. Math. 31, 2006, 463-478.

[9] Halburd, R. G., and R. J. Korhonen: Finite-order meromorphic solutions and the discrete Painlevé equations. - Proc. Lond. Math. Soc. (3) 94:2, 2007, 443-474.

[10] Halburd, R. G., R. J. Korhonen, and K. Tohge: Holomorphic curves with shift-invariant hyperplane preimages. - Trans. Amer. Math. Soc. 366, 2014, 4267-4298.

[11] Hayman, W. K.: Meromorphic functions. - Oxford Math. Monogr., Clarendon Press, Oxford, 1964.

[12] Hayman, W. K.: Warings Problem für analytische Funktionen. - Bayer. Akad. Wiss. Math. Natur. Kl. Sitzungsber. 1984 (Bayer. Akad. Wiss., Munich), 1985, 1-13.

[13] IYeR, G.: On certain functional equations. - J. Indian Math. Soc. 3, 1939, 312-315. 
[14] LiU, K., and T. B. CAO: Entire solutions of Fermat type q-difference differential equations. Electron. J. Differential Equations 2013:59, 2013, 1-10.

[15] Liu, K., T. B. CAO, and H.Z. CAO: Entire solutions of Fermat type differential-difference equations. - Arch. Math. (Basel) 99:2, 2012, 147-155.

[16] Laine, I.: Nevanlinna theory and complex differential equations. - de Gruyter Stud. Math. 15, de Gruyter, Berlin, 1993.

[17] Milne-Thomson, L. M.: The calculus of finite differences. - Amer. Math. Soc., 2000.

[18] Newman, D. J., and M. Slater: Waring's problem for the ring of polynomials. - J. Number Theory 11, 1979, 477-487.

[19] TAYlor, R., and A. Wiles: Ring-theoretic properties of certain Hecke algebras. - Ann. of Math. (2) 141, 1995, 553-572.

[20] Toda, N.: On the functional equation $\sum_{i=0}^{p} a_{i} f_{i}^{n_{i}}=1$. - Tôhoku Math. J. 23, 1971, 289-299.

[21] Wiles, A.: Modular elliptic curves and Fermat's last theorem. - Ann. of Math. (2), 141, 1995, $443-551$.

[22] Yang, L.: Value distribution theory. - Springer-Verlag, Berlin, 1993.

[23] YAnG, L.Z., and J.L. Zhang: Non-existence of meromorphic solutions of a Fermat type functional equation. - Aequationes Math. 76:1-2, 2008, 140-150.

[24] YI, H. X., and C. C. YANG: Uniqueness theory of meromorphic functions. - Kluwer Academic Publishers, 2003.

[25] YI, H. X., and L. Z. YANG: On meromorphic solutions of Fermat type functional equations. Sci. China Ser. A 41:10, 2011, 907-932 (in Chinese).

Received 1 December 2014 • Accepted 30 March 2015 